\title{
High level of estradiol in a Holstein-Friesian cow with sacroiliac luxation after calving
}

\author{
Lovelia L. Mamuad ${ }^{1}$, Bosung Seo ${ }^{2}$, Seogjin Kang ${ }^{2}$, Yong-Il Cho ${ }^{1, *}$ \\ ${ }^{\prime}$ Department of Animal Science and Technology, College of Bio-industry Science, Sunchon National University, \\ Suncheon 57922, Korea \\ ${ }^{2}$ Department of Animal Resources Development, National Institute of Animal Science, Rural Development Administrator \\ Cheonan 31000, Korea
}

Abstract: This is the report of sacroiliac luxation in a Holstein-Friesian cow, describing the progression of the sacroiliac luxation from the onset of its development with changes in estradiol $\left(\mathrm{E}_{2}\right)$ and serum chemistry. The high estrogen concentration was assumed to be a predisposing factor of the disease that relaxed the pelvic muscles and a secondary slippery finally caused the sacroiliac luxation. The $\mathrm{E}_{2}$ was present at levels four times higher in the cow with sacroiliac luxation $(167 \mathrm{pg} / \mathrm{mL})$ than in normal cows. Above normal levels of creatinine kinase, aspartate aminotransferase, and lactate dehydrogenase were also observed in cow with sacroiliac luxation.

Keywords: estradiol, Holstein-Friesian cow, sacroiliac luxation

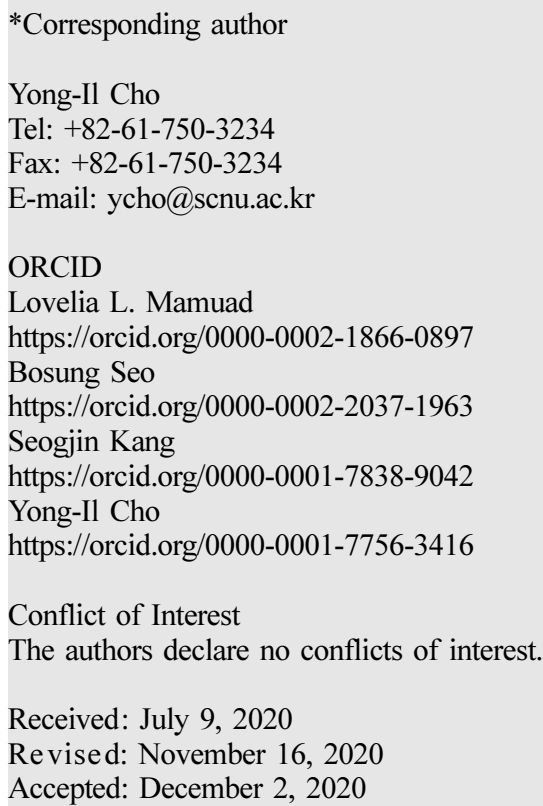

Sacroiliac luxation or subluxation is a musculoskeletal disease in animals defined as partial to complete separation of the fibrocartilaginous sacroiliac joint surfaces [1]. This happens when the ligamentous attachment at the junction of the pelvis and the sacrum relaxes [2] or the joint is dislocated and the pelvis is no longer connected to the sacrum [3]. Dystocia of a fetal origin or accidents is the predisposing factors that sacroiliac luxation is generally associated with [4]. Dropping of the spine in sacroiliac luxation may develop over two to three days [5]; however, sacroiliac luxation in cattle has rarely been reported when compared to cats and dogs. This condition occurs around the periparturient period and is characterized by the back between the sacral tuberosities appearing to have dropped and become more prominent. However, there have been no reports of changes in hormones, serum chemistry, or clinical history as the disease progressed in cattle. Therefore, this study was conducted to provide diagnostic indicators related to the causes of the disease and guidelines for making future decisions (e.g., treatment or culling) regarding cattle.

A Holstein-Friesian cow was 3 years old, approximately $540 \mathrm{~kg}$ body weight, and had first calving. During calving, the cow showed weak dystocia and partially detached placenta. The cow had difficulty while calving because the calf was in the posterior presentation. However, with veterinary assistance, the calf was delivered as stillborn.

On the fifth day after calving, the cow fell down several times on a slippery surface on its way out from the milking parlor after morning milking because of heavy rain. The cow was referred to a veterinarian when it was unable to rise. The body temperature and ketone level of the cow were $39.4^{\circ} \mathrm{C}$ and $0.3 \mathrm{mM} / \mathrm{L}$, respectively. With the help of farmers and to prevent secondary injury, the cow was moved to an unpaved area. While it was being moved, the cow staggered to its feet. The cow was also growling in pain while it was sitting, but this stopped when it stood up. To relieve the cow's pain, a non-steroid anti-inflammatory drug (NSAID; $10 \mathrm{~mL}$ ketoprofen $[100 \mathrm{mg} / \mathrm{mL}]$ ) was injected as intramuscular (IM). At this point, the cow was diagnosed with sacroiliac luxation as a result of its physical status (Fig. 1).

The next day (day 6), the cow tried to stand up but continuously slipped and fell on the soil floor. Although the cow had a good appetite, it was still 
Day 5

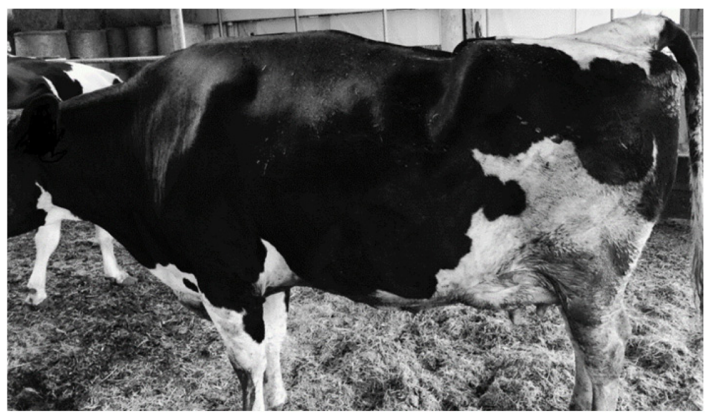

Day 11

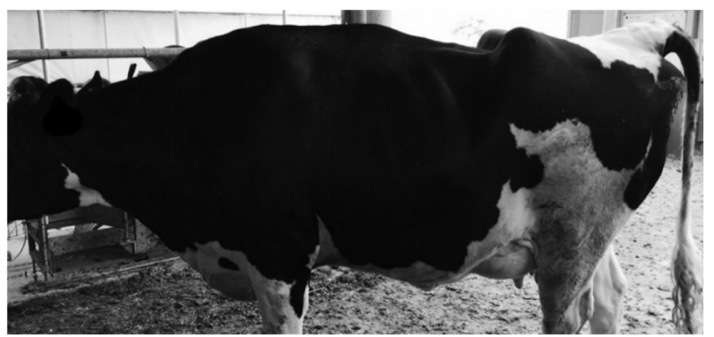

Day 8

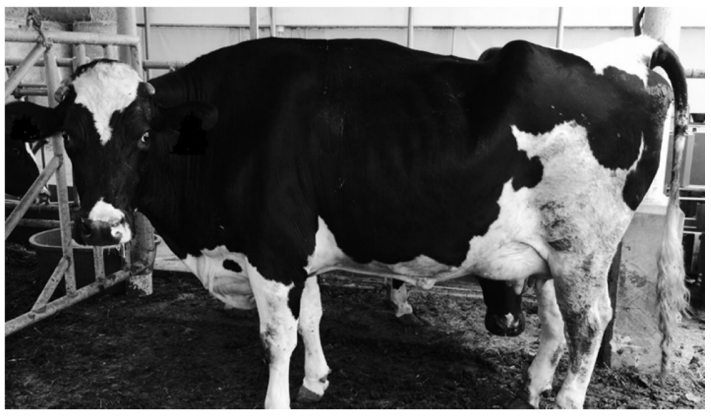

Day 16

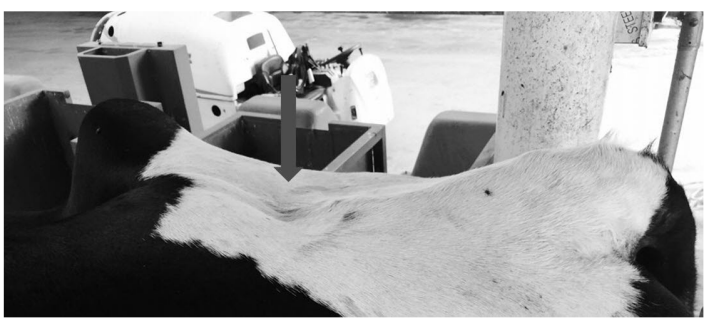

Fig. 1. Development of sacroiliac luxation in the cow after onset of the disease. The arrow in day 16 indicates the tuber coxae insertion.

growling in pain; hence, the $10 \mathrm{ml}$ ketoprofen $[100 \mathrm{mg} / \mathrm{mL}]$ was again injected as IM. At this time, the body temperature, ketone, and glucose level of the cow were $39.4^{\circ} \mathrm{C}, 0.6$ $\mathrm{mM} / \mathrm{L}$, and $74 \mathrm{mg} / \mathrm{dL}$, respectively. On day 7 , the cow could stand by itself from sitting and showed good appetite, indicating the NSAID relieved the pain in the cow. On day 11 after calving, we found that the condition of the cow had become worse while walking. The hind legs were twisted, which made it even more difficult for the cow to step forward on its right leg on day 13. The cow continually maintained its good appetite until day 16 after calving; however, the condition became worse with time. Moreover, slight insertion of the posterior part of the tuber coxae was observed on day 16 after calving (Fig. 2). The sacral area of the cow appeared to be dropped and the tuber coxae were very pronounced.

Blood samples were collected from the jugular vein of the cow from day 5 to 14 after calving to further evaluate the clinical signs and enable diagnosis. The collected blood sample was transferred into collection tubes (vacutainer SST tubes [Becton Dickinson, USA]). Blood chemistry (Creatinine kinase, aspartate aminotransferase, and lactate dehydrogenase) was analyzed using an an IDEXX Catalyst One Chemistry Analyzer. The estradiol $\left(\mathrm{E}_{2}\right)$ concentration was analyzed using an Immulite 1000 system enzyme-amplified chemiluminescence with an analytical sensitivity of $15 \mathrm{pg} /$ $\mathrm{mL}$. Briefly, the blood samples were ultracentrifuge to clear lipemic samples. Then, the samples were incubated as well as the reagent together with the coated bead for $60 \mathrm{~min}$.
Finally, the chemiluminescent substrate was added to the test unit containing the bead and the signal is generated in proportion to the bound enzyme.

The average $E_{2}$ level of the cow with sacroiliac luxation was four times greater (approximately $167 \mathrm{pg} / \mathrm{mL}$ ) than that of six randomly selected normal cows at different reproductive status having an average of $41.4 \mathrm{pg} / \mathrm{mL}$ within the same barn (Fig. 3). After the cow was diagnosed, the high $\mathrm{E}_{2}$ concentration continued for several days with $105 \mathrm{pg} / \mathrm{mL}$ and $229 \mathrm{pg} / \mathrm{mL}$ at day 8 and 10, respectively. Similarly, creatinine kinase (CK), aspartate aminotransferase (AST) and lactate dehydrogenase (LDH) levels were above the normal range. The highest CK concentration was observed on day 6 after calving and this became lower with time, reaching normal levels on day 14 after calving. The level of AST was highest on day 8 , and then gradually declined, while the LDH level was highest on day 6 , then gradually decreased. However, both the AST and LDH level were maintained at above the normal range until day 14 (Fig. 3).

Sacroiliac displacement etiology usually involves excessive ligamentous flaccidity around parturition, wherein hemorrhage and soft tissue damage occurs in the joint space and peripherally [5]. The cow, in this case, was observed to have tuber coxa that raised high above the lumbar vertebra and the gait posture of the hind limbs was rather unstable, resulting in a swing back and forth when the cattle walking, which is a sign of luxation [6]. Unlike in small animals like cats and dogs, sacroiliac luxation in cows is very rare [7] and surgical treatments cannot be conducted [3]. Although cows diag- 


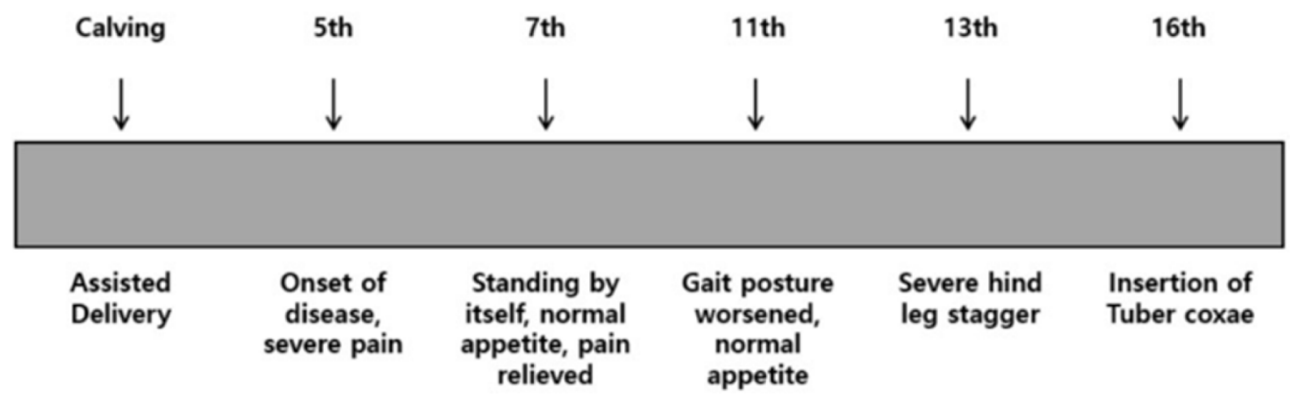

Fig. 2. Timeline of the physical status of the cow as sacroiliac luxation progressed.
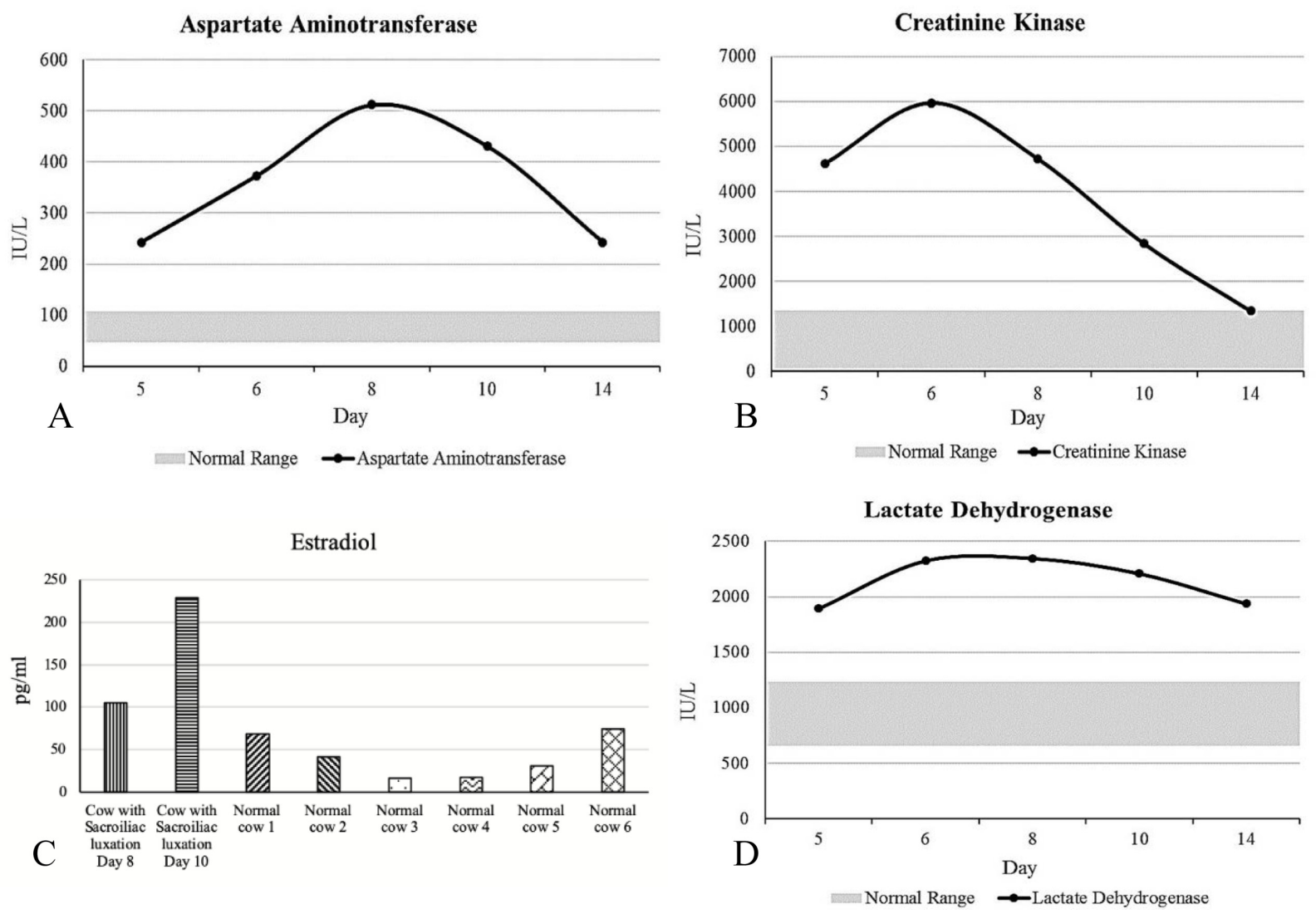

Fig. 3. Change in estradiol $\left(\mathrm{E}_{2}\right)$ and serum chemistry (A: aspartate aminotransferase, B: creatinine kinase, C: estradiol, D: lactate dehydrogenase) of the cow.

nosed with sacroiliac luxation may be able to survive for years with a well-bedded stall, the prognosis is very poor because of gait disorder, high risk of mastitis infection, the absence of mounting, low conception rate, and dystocia in next calving.

This is the first report to describe the progression of sacroiliac luxation from the onset of its development with the changes in hormones $\left(\mathrm{E}_{2}\right)$, serum chemistry, electrolytes, and clinical history. The cow was growling in severe pain for hours and the spine dropped. Even though the cow maintained a good appetite, its gait posture became worse and it was unlikely to recover to a normal stance and gait. This cattle showing sacroiliac luxation was recommended to be slaughtered as soon as possible rather than receiving treatment. Normally, the plasma $\mathrm{E}_{2}$ concentrations gradually increased from gestation day to prepartum. In the study of Lammoglia et al. [8], the $\mathrm{E}_{2}$ concentrations ranged from $5 \mathrm{pg} /$ $\mathrm{mL}$ to $55 \mathrm{pg} / \mathrm{mL}$ in the induced and synchronized estrus in cattle that is similar to the results we obtained in the normal cows in this study. Also, the plasma $\mathrm{E}_{2}$ concentrations and sacrosciatic ligament relaxation observed in the study are positively correlated to each other wherein they increased gradually as gestation advanced and reached the peak level on the day before calving and decreased after calving [9]. Moreover, the average $\mathrm{E}_{2}$ levels were four times higher than in normal cows without any metabolic diseases (i.e., hypocal- 
cemia, hypomagnesemia, and ketosis) in this study. This finding was similar to the previous report that peak estrogen level associated with sacroiliac luxation or subluxation [6]. The CK, AST, and LDH levels were also above the normal range, which were a result of muscle injury caused by sacroiliac luxation of the cow when it fell on the slippery ground. However, both the calcium and phosphorous levels were within the normal range (data not shown). Therefore, the high estrogen concentration and dystocia were presumed to be predisposing factors of the disease based on relaxing of the pelvic muscles and the second fall that caused the sacroiliac luxation in this study.

\section{Acknowledgments}

The study was supported by a grant from the Sunchon National University Research Fund in 2020(2020-0230) and the Cooperative Research Program for Agriculture Science and Technology Development (PJ01269704) funded by the Rural Development Administration, Republic of Korea.

\section{References}

1. Scott PR, Penny CD, Macrae AI. Sacroiliac luxation/ subluxation. Musculoskeletal diseases chapter. In: Northcott J (ed.). Cattle Medicine. pp. 186-187, Manson Publishing Ltd., London, 2011.
2. Blowey R, Weaver DA. Locomotor disorders. In: Color Atlas of Diseases and Disorders of Cattle E-Book. 3rd ed. pp. 127-128, Mosby Elsevier Ltd., Maryland Heights, 2011.

3. Rochat MC. Sacroiliac luxation. musculoskeletal section. In: Morgan R (ed.). Small Animal Practice Client Handouts. p. 300, Saunders Elsevier, Philadelphia, 2011.

4. Afonso JA, Souto RJ, Filho AP, Coutinho LT, De Mendonça CL, De Azevêdo Costa N. Sacroiliac luxation in cow: case report. Rev Bras Med Vet 2011;33:29-31.

5. Andrews AH, Blowey RW, Boyd H, Eddy RG. Bovine Medicine: Diseases and Husbandry of Cattle. 2nd ed. pp. 447-448, Blackwell Science Ltd., Oxford, 2004.

6. Divers TJ, Peek SF. Musculoskeletal disorders. In: Rebhun's Diseases of Dairy Cattle. 3rd ed. pp. 496-497, Saunders Elsevier, Philadelphia, 2008.

7. Schlicksup MD, Holt DE, Mai W, Holmes ES, Agnello KA. The effect of abaxial retraction on pelvic geometry after pelvic symphysiotomy. Vet Surg 2013;42:958-962.

8. Lammoglia MA, Short RE, Bellows SE, Bellows RA, MacNeil MD, Hafs HD. Induced and synchronized estrus in cattle: dose titration of estradiol benzoate in peripubertal heifers and postpartum cows after treatment with an intravaginal progesterone-releasing insert and prostaglandin F2 $\alpha$. J Anim Sci 1998;76:1662-1670.

9. Shah KD, Nakao T, Kubota H. Plasma estrone sulphate (E1S) and estradiol-17 $\beta$ (E2 $\beta$ ) profiles during pregnancy and their relationship with the relaxation of sacrosciatic ligament, and prediction of calving time in Holstein-Friesian cattle. Anim Reprod Sci 2006;95:38-53. 